\title{
LeE SANG GaK TELeScope (LSGT): A Remotely Operated Robotic Telescope For EDUCATION AND RESEARCH AT SEOUl National University
}

\author{
Myungshin Im ${ }^{1,2,3}$, Changsu Choi ${ }^{1,2}$, And Kinyun Kim ${ }^{2}$ \\ ${ }^{1}$ Center for the Exploration of the Origin of the Universe, Department of Physics and Astronomy, Seoul National \\ University, Gwanak-gu, Seoul 151-742, Korea; mim@astro.snu.ac.kr \\ ${ }^{2}$ Astronomy Program, Department of Physics and Astronomy, Seoul National University, Gwanak-gu, Seoul 151-742, Korea \\ ${ }^{3}$ Korea Institute for Advanced Study, 85 Hoegiro, Dongdaemun-gu, Seoul 130-722, Republic of Korea
}

Received July 3, 2015; accepted July 31, 2015

\begin{abstract}
We introduce the Lee Sang Gak Telescope (LSGT), a remotely operated, robotic 0.43-meter telescope. The telescope was installed at the Siding Spring Observatory, Australia, in 2014 October, to secure regular and exclusive access to the dark sky and excellent atmospheric conditions in the southern hemisphere from the Seoul National University (SNU) campus. Here, we describe the LSGT system and its performance, present example images from early observations, and discuss a future plan to upgrade the system. The use of the telescope includes (i) long-term monitoring observations of nearby galaxies, active galactic nuclei, and supernovae; (ii) rapid follow-up observations of transients such as gamma-ray bursts and gravitational wave sources; and (iii) observations for educational activities at SNU. Based on observations performed so far, we find that the telescope is capable of providing images to a depth of $R=21.5 \mathrm{mag}$ (point source detection) at 5- $\sigma$ with 15 min total integration time under good observing conditions.
\end{abstract}

Key words: telescopes — instrumentation: detectors — methods: observational — technique: photometric

\section{INTRODUCTION}

To obtain high quality data for astronomical research, it is necessary to install telescopes at locations offering dark sky, low humidity, and stable atmospheric conditions. Such places are usually found in remote locations, on mountain-tops that do not allow easy access, forcing astronomers to make long trips to these locations and making it difficult to carry out observations flexibly such as a rapid observation of new interesting astronomical sources. However, recent advances in computer networking and remote control technologies have made it possible to operate telescopes remotely from places such as cities where most astronomers reside. Nowadays, professional astronomers often carry out observations from a remote location (e.g., their office). Amateur astronomers are also setting up remotely operated telescopes in locations favorable for astronomical observations.

Seoul National University (SNU) has several optical telescopes on campus that are equipped with optical imagers and a low resolution spectrograph. These telescopes have been used for educational activities of SNU students and occasionally for research projects. However, the use of these telescopes has suffered greatly from light-pollution due the fast development of the metropolitan Seoul area in the last several decades. The sky brightness at SNU has been reported as $B \simeq 17-$ $18 \mathrm{mag} \operatorname{arcsec}^{-2}$ and $V \simeq 16-17.3 \mathrm{mag} \operatorname{arcsec}^{-2}$ (Lee

Corresponding AUthor: M. Im et al. 2009), several hundred times brighter than the night sky of astronomical observatories at remote locations (e.g., Leinert \& Mattila 1998; Patat 2003; Pedani 2009; Aceituno et al. 2011). The bright night sky limits observations at the SNU campus to only bright objects with $R \lesssim 18.3 \mathrm{mag}$ with a 0.6 -meter telescope (Choi et al. $2014 ; 5-\sigma$ detection limit of a point source at $15 \mathrm{~min}$ total integration).

In order to access dark skies with excellent astroclimate, we have installed instruments on telescopes at several remote sites. Examples include the Seoul National University $4 \mathrm{k} \times 4 \mathrm{k}$ Camera (SNUCAM) on the 1.5-meter telescope of the Maidanak observatory, Uzbekistan (Im et al. 2010), and the Camera for QUasars in EArly uNiverse (CQUEAN) of the 2.1meter telescope at the McDonald observatory (Park et al. 2010; Kim et al. 2010; Lim et al. 2013). However, the use of these facilities has been rather limited, mostly due to high demands for the telescopes for other research projects. In order to gain regular, exclusive access of the southern hemisphere dark sky, we recently installed a remotely operated, robotic telescope at the Siding Spring Observatory (SSO) in Australia. We named this telescope as "Lee Sang Gak Telescope" (LSGT) to honor the recently retired SNU professor who donated a significant portion of the funding that made this telescope possible. LSGT is now regularly used for observational classes and research projects at SNU.

LSGT is anticipated to be extensively used by both 


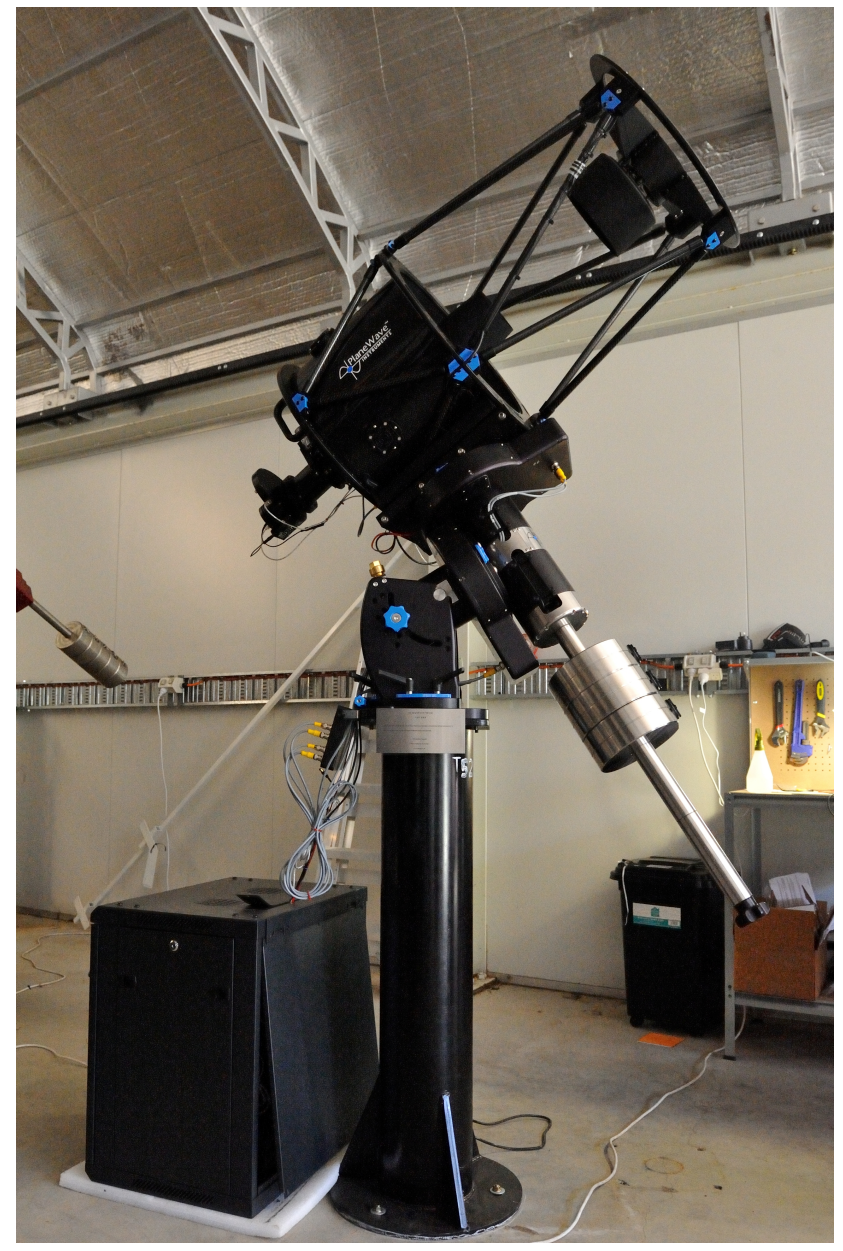

Figure 1. The Lee Sang Gak Telescope, shown shortly after its installation at the Siding Spring Observatory, Australia in 2014 October.

astronomers and students. To help the potential telescope users to plan observations with LSGT, we describe in this paper the overall characteristics of LSGT, its performance, and highlights from its usage so far.

\section{SySTEM}

LSGT (Figure 1) is a Corrected Dall-Kirkham (CDK) design telescope with a 0.43 -meter (17-inch) diameter primary mirror. It is manufactured by the PlaneWave Instruments $^{\mathbf{1}}$ and matched with a PlaneWave Instrument's Ascension 200HR mount. The CDK design adopts an ellipsoidal primary mirror, a spherical secondary mirror, and a combination of two lenses to produce a coma-free flat field with no off-axis astigmatism over a $52 \mathrm{~mm}$ diameter circle. Given the good performance of the mount and a need to rapidly observe targets, we opted not to use an auto-guiding system. The effective focal ratio of the telescope is $\mathrm{f} / 6.8$, and the telescope is designed to achieve a good, distortion-free image quality over 1 degree diameter circle. Figure 2 shows the combined throughput of the reflectivity of

\footnotetext{
$\mathbf{1}_{\text {http: }} / /$ planewave.com
}

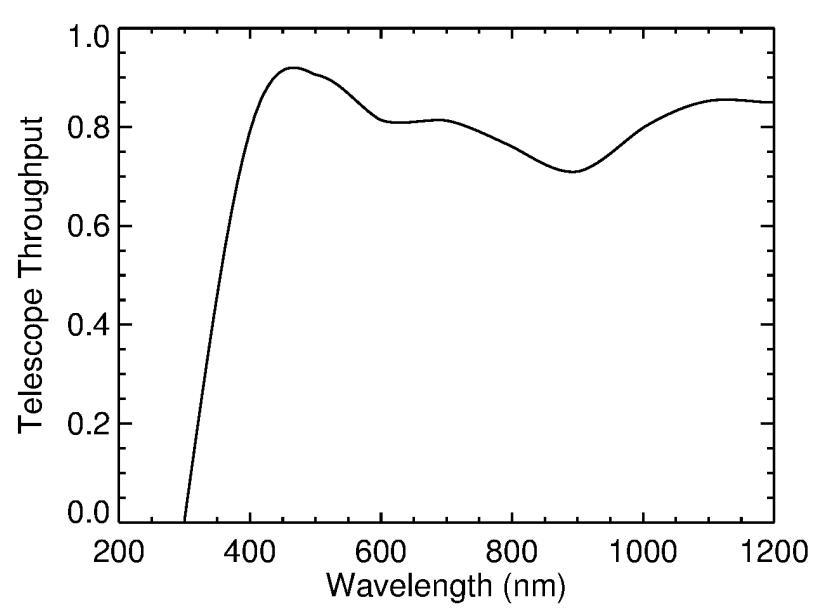

Figure 2. The overall throughput of the optical telescope system of LSGT.

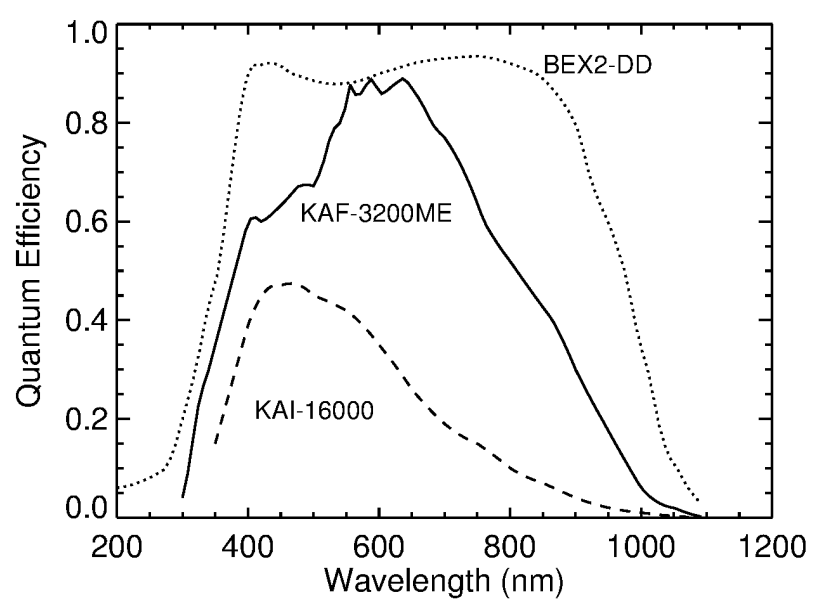

Figure 3. QEs of the CCDs on different cameras that have been in use or planned for LSGT. The current camera uses the KAF-3200ME chip (solid line), while we plan to upgrade it with a new camera that is equipped with a deep-depletion chip (dotted line). The data are taken from the manufacturer's data sheet.

the mirrors and the transmittance of the lenses. The data are kindly provided by PlaneWave Instruments.

The currently available instrument is the ST-10XME camera of the SBIG Astronomical Instruments (a division of Diffraction Limited). This camera uses the KAF-3200ME chip, which offers a peak quantum efficiency (QE) of $85 \%$ and a pixel size of $6.8 \mu \mathrm{m}$ in a 2184 $\mathrm{x} 1472$ layout. On LSGT, the pixel scale translates to 0 .'48, and a field of view of $17 ! 5 \times 11 ! 8$. Another camera, Starlight Xpress's SXVR-H36, is also available on demand. The CCD chip, the Truesense's KAI-16000 interline CCD, has a dimension of $36.3 \mathrm{~mm} \times 24.4 \mathrm{~mm}$ $(4904 \times 3280$ pixels $)$ with a physical pixel size of 7.4 $\mu \mathrm{m}$. On LSGT, it gives a pixel scale of 0.52 and a field of view of $32.7 \times 26.2$. The QEs of these two cameras, along with a planned future upgrade (Section 6), are plotted in Figure 3. 


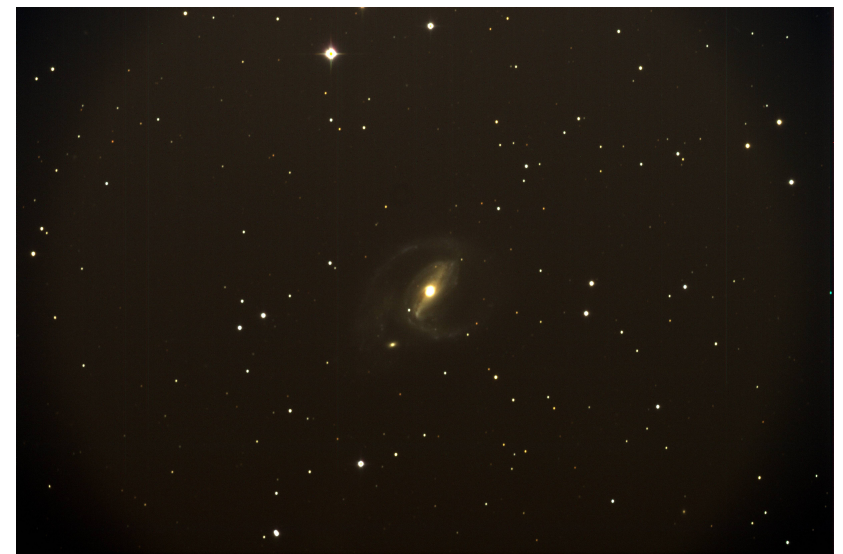

Figure 4. A $B V R$ composite color image of NGC 1097 taken with the SXVR-H36 camera. While the image quality is good over the whole field, vignetting is visible near the corners of the image.

Table 1 summarizes the characteristics of the cameras on LSGT. The standard $U B V R I$ and luminance, $\mathrm{H} \alpha$, $\mathrm{S}$ II, and O III filters are currently available. The telescope is installed in a roll-off-roof dome, together with other telescopes that are managed by iTelescope.Net Pty. Ltd. ${ }^{2}$. The management of the telescope by the private company saves efforts of SNU personnel.

\section{Operation and Data Reduction}

Observations have been carried out either robotically by writing a "plan" script that lists a number of commands that specify the observation of a target, or by typing in observational commands remotely in real-time. Under the robotic operation, the observations are executed at the time specified in the plan automatically. In both cases, we access the system through a standard web browser system that is set up by iTelescope.Net.

The focusing of the telescope is done by running an auto-focus program over a target field or a field with bright stars near the target. The typical overhead for the auto-focusing is about $\sim 3$ minutes, including the telescope slew time. The command scripts allow us to skip focusing through different filters by adopting a pre-defined focus offset, and to specify the frequency of the focusing. Typical observations require focusing approximately every two hours. Bias and dark frames are taken regularly during daytime or cloudy nights with the cover of the telescope and the dome closed. The flats are taken on demand several times per month using a flat-field screen. The flat-field pattern shows little variation day-by-day so the sparse acquisition of the flat field image is adequate. As soon as the data are taken, the system performs the bias/dark subtraction and flatfielding with a standard set of calibration files. The raw images as well as the calibrated images become available soon after the observation (typically a few minutes after) for download.

\footnotetext{
${ }^{2}$ http://itelescope.net
}

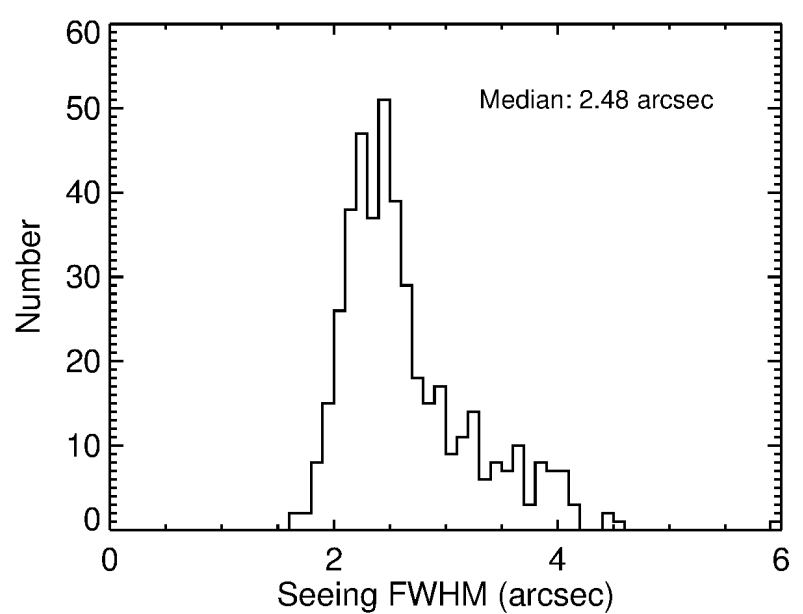

Figure 5. The $R$-band seeing statistics between 2014 November 26 through 2015 April 17. The median seeing is 2. . 48 .

\section{Performance}

Since the installation of LSGT at SSO in 2014 October, we have been improving the system performance. Here, we report the current performance of the telescope based on observational data.

\subsection{SXVR-H36}

Early test observations in 2014 October and November were carried out using the SXVR-H36 camera. Figure 4 shows a test image of NGC 1097 taken with this camera. $B, V$, and $R$ band images were combined to create the composite image with $300 \mathrm{sec}$ exposure per band. Basic calibration of bias and dark subtraction and flat-fielding was done. The overall quality of the image is good, but a vignetting pattern can be seen near the corners of the chip. From the image, we measure a $5-\sigma$ limiting magnitude for point sources is $V=20$ mag with $300 \mathrm{sec}$ exposure at $3{ }^{\prime \prime} 0$ seeing and a dark night. The optical alignment was not optimal during the test observations with the SXVR-H36 camera, but it allowed a uniform image quality over the camera field of view with the $\sim 33^{\prime \prime} 0$ seeing.

\subsection{SBIG ST-10XME and Limiting Magnitude}

Since 2014 November 14, LSGT observations have been carried out using the ST-10XME camera. We decided to use the ST-10XME camera for regular operations partly due to the better sensitivity of the camera in comparison to the SXVR-H36 camera, and due to the availability of a filter wheel that can house ten filters.

Figure 5 summarizes the seeing values for the $R$-band images taken during 2014 November 26 through 2015 April 17. The optical alignment has been improved during this period of operation, and we sometimes achieved seeing conditions of 1 .' 8 . With the ST-10XME camera, we achieved limiting magnitudes of $R=21.5 \mathrm{mag}$ for a 15 min exposure, with a clear dark night and a seeing size of 2.2 arcsec. 
Table 1

LSGT Camera Specifications

\begin{tabular}{lcccccccc}
\hline Camera & $\begin{array}{c}\text { Pixel scale } \\
\operatorname{arcsec}\end{array}$ & $\begin{array}{c}\text { Field of view } \\
\operatorname{arcmin}^{2}\end{array}$ & $\begin{array}{c}\text { RN } \\
\mathrm{e}^{-}\end{array}$ & $\begin{array}{c}\text { Dark current } \\
\mathrm{e}^{-} / \mathrm{pix} / \mathrm{sec}\end{array}$ & Peak QE & $\begin{array}{c}\text { Gain } \\
\mathrm{e}^{-} / \mathrm{DN}\end{array}$ & $\begin{array}{c}\text { Full well } \\
\mathrm{e}^{-}\end{array}$ & $\begin{array}{c}\text { Readout time } \\
\text { sec }\end{array}$ \\
\hline ST-10XME $^{\mathrm{a}}$ & 0.48 & $17.5 \times 11.8$ & $10.0^{\mathrm{b}}$ & $0.01-0.5^{\mathrm{b}, \mathrm{c}}$ & 0.85 & $1.42^{\mathrm{b}}$ & $\sim 78,000^{\mathrm{b}}$ & $\sim 20^{\mathrm{b}}$ \\
SXVR-H36 & 0.52 & $32.7 \times 26.2$ & $9^{\mathrm{d}}$ & $0.01^{\mathrm{d}}$ & 0.45 & $0.4^{\mathrm{d}}$ & $30,000^{\mathrm{d}}$ & $\sim 10$ \\
\hline
\end{tabular}

a Operating camera as of 2015 August 31.

b Measurements with CCD temperature at $-10^{\circ} \mathrm{C}$.

${ }^{\mathrm{c}}$ For most of the pixels, $<0.05 \mathrm{e}^{-} / \mathrm{pix} / \mathrm{sec}$. But, $6 \%$ are warm pixels with $0.05-0.5 \mathrm{e}^{-} / \mathrm{pix} / \mathrm{sec}$ and $2 \%$ with $>0.5 \mathrm{e}^{-} / \mathrm{pix} / \mathrm{sec}$.

d Manufacturer's data.

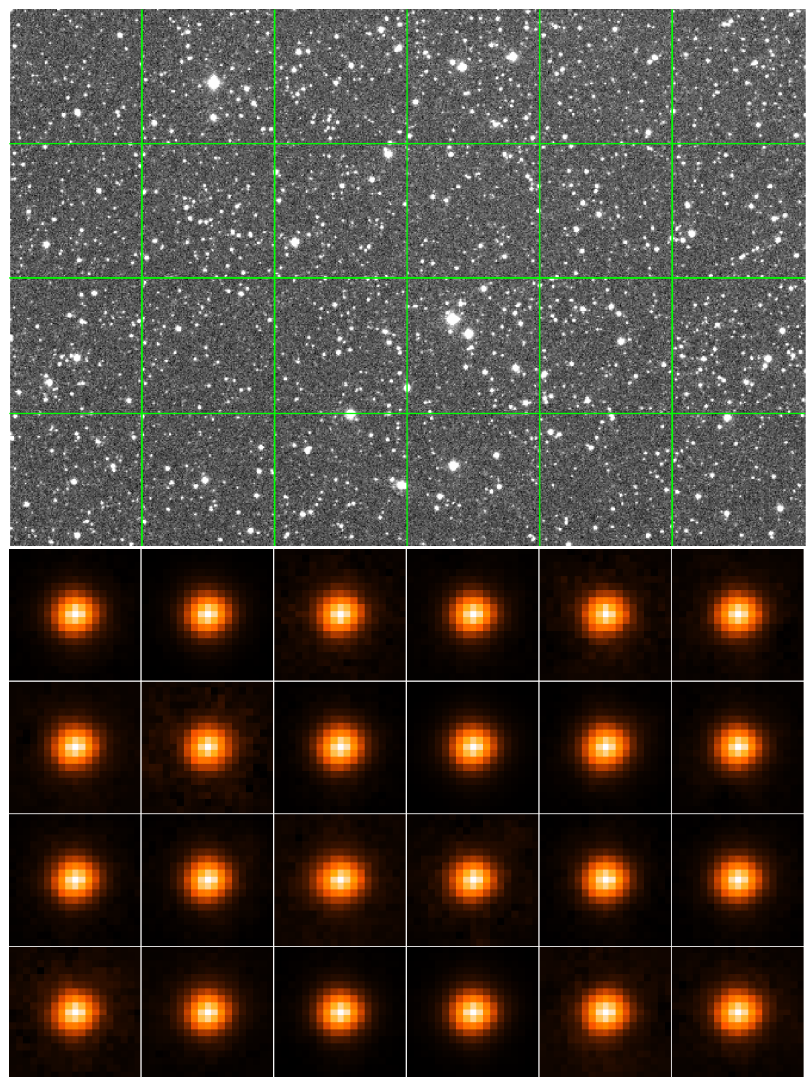

Figure 6. The PSF image at different sections of the field of view of the ST-10XME camera. The PSF shape is uniform over the camera field of view, but is slightly elongated, which is partly due to the inaccuracy in tracking, but mostly due to the telescope optics.

\subsection{Optical Performance and Tracking Accuracy}

In Figure 6, we show the $R$-band images of the point spread function (PSF) at different parts of the field of view. The PSFs are constructed using stars in each section of the image. An exposure time of $60 \mathrm{sec}$ is used for this image. We find that the image quality is uniform over the field of view of ST-10XME with a PSF FWHM of 2. . 2 . However, stars appear slightly elongated (an axis ratio, $b / a$, of $\sim 0.95)$. The PSF elongation is also visible in the images taken with much shorter exposures (5 sec). Therefore, we conclude that the elongation is caused mainly by the optics of the telescope.

Additionally, we note that the PSF can be elongated due to the inaccuracy in tracking for long exposure times. We examined the tracking accuracy using a series of images taken over a long period of time. If the tracking is perfect, we expect to find that a star will be located at the same position on the chip in successive frames. Otherwise, the star will move toward a certain direction in successive exposures. We find a drift of about $0.22 / \mathrm{min}$ along both the RA and Dec directions, with the direction of the drift changing depending on the location on the sky. This test indicates a total drift of $\sim 0.3 / \mathrm{min}$.

We calculate how this kind of tracking error elongates the PSF. Let us assume that the telescope pointing drifts toward a single direction at a rate of $k$ in units of $\operatorname{arcsec} / \mathrm{min}$. For a given exposure time $t_{\text {exp }}$, this translates into a movement of a star position by an amount of $k t_{\text {exp }}$ arcmin, which causes an elongation of the stellar image in that direction. Assuming that the PSF profile, $\operatorname{PSF}(x)$, can be approximated with a Gaussian function, the elongated PSF profile can be described as,

$$
\begin{aligned}
& \operatorname{PSF}(x) \sim \frac{1}{\sqrt{2} \sigma} \int_{-t_{\text {exp }} / 2}^{t_{\text {exp }} / 2} \exp \left[-\frac{(x-k t)^{2}}{2 \sigma^{2}}\right] \frac{\mathrm{d} t}{t_{\text {exp }}} \\
& \propto \operatorname{erf}\left(\frac{0.5 k t_{\text {exp }}-x}{\sqrt{2} \sigma}\right)-\operatorname{erf}\left(\frac{-0.5 k t_{\text {exp }}-x}{\sqrt{2} \sigma}\right),
\end{aligned}
$$

where $\sigma=\mathrm{FWHM} / 2.35$, and $x$-direction is taken as the direction of the elongation. The resulting function resembles the Gaussian form closely, and the FWHM and $b / a$ values of the elongated PSF can be derived numerically using Equation (1).

In Figure 7, we show how the axis ratio changes a function of $t_{\text {exp }}$ according to Eq. (1). The current system hardly achieves a PSF FWHM better than 2'. 0 . Under such conditions, a $180 \mathrm{sec}$ exposure can create an elongated $\mathrm{PSF}$ with $b / a \simeq 0.95$. For seeing values of FWHM $\sim 3$.' $^{\prime}$, the PSF elongation is not noticeable even with an exposure of around $5 \mathrm{~min}$. We conclude that the current system can be used to take images with $180 \mathrm{sec}$ exposure, without distorting the PSF shape worse than $b / a=0.95$.

\section{Science Programs and Current Usage}

Currently, there is one regular science program running on the telescope. It is the Intensive Monitoring 


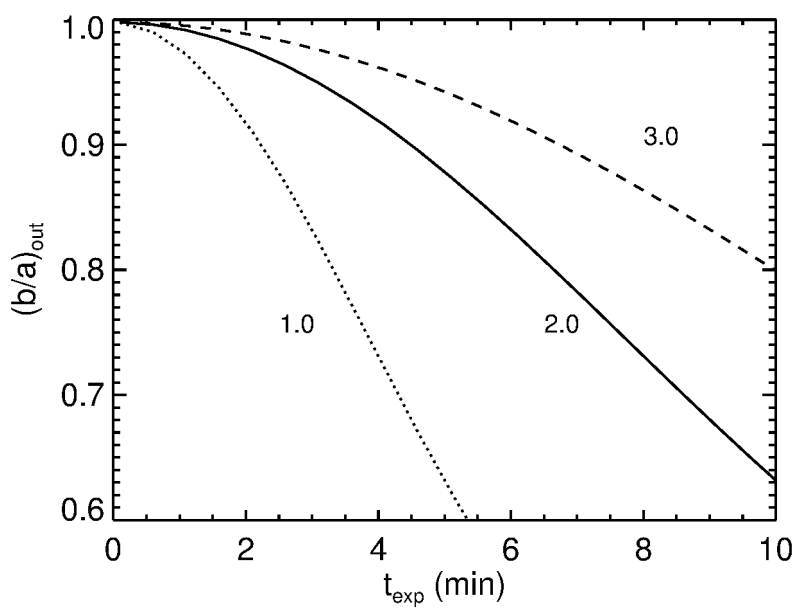

Figure 7. The PSF elongation (axis ratio) due to a drift in the telescope pointing as a function of the exposure time $\left(t_{\text {exp }}\right)$. The numbers next to each line indicate the FWHM (arcsec) of a round PSF without the pointing drift. The drift rate is assumed to be $0 .{ }^{\prime \prime} 3 / \mathrm{min}$.

\section{Table 2}

Current LSGT Performance

\begin{tabular}{lcc}
\hline Limiting magnitude & Seeing & Tracking accuracy \\
\hline$R=20.5^{a}-21.5^{b, c}$ & $2^{\prime \prime}-3^{\prime \prime}$ & $0^{\prime \prime} \cdot 3 / \mathrm{min}$ \\
\hline
\end{tabular}

a 5 - $\sigma$ detection of point source with 15 min exposure, $2^{\prime \prime} .2$ seeing, and full moon.

b Same as above, but with new moon.

c The limiting magnitudes in $B$ and $V$ (under new moon) are about the same as the $R$-band value.

Survey of Nearby Galaxies (IMSNG). IMSNG performs high cadence imaging observations of nearby galaxies using facilities at several different observatories. By using telescopes at multiple time zones, it is possible to achieve very high cadence monitoring of nearby galaxies with time intervals as short as 2-3 hours. Since 2014 December, we have been using LSGT to monitor nearby galaxies at distances less than $50 \mathrm{Mpc}$. These galaxies are chosen to have high near-ultraviolet (NUV) fluxes, implying high star formation rates. The scientific aim of the project is to detect transients, such as supernovae, in a very early phase to gain insights about their energetics. Through this program, we succeeded in detecting SN $2015 \mathrm{~F}$ in a very early phase at a daily cadence (Im et al. 2015). Figure 8 shows NGC 2442 before and after the appearance of SN $2015 \mathrm{~F}$.

We have also taken other verification images to evaluate the performance of LSGT. In Figure 9, we show single frame images of NGC 2467 (a star forming region), NGC 2207 (an interacting galaxy pair), and a mosaic image of Omega Centauri (NGC 5139). During SNU observational classes for astronomy majors, LSGT has been used to study brightness variations of asteroids, color-magnitude diagrams of stellar clusters, surface brightness profiles of nearby galaxies, and light curves of variable stars and exoplanet transit events.

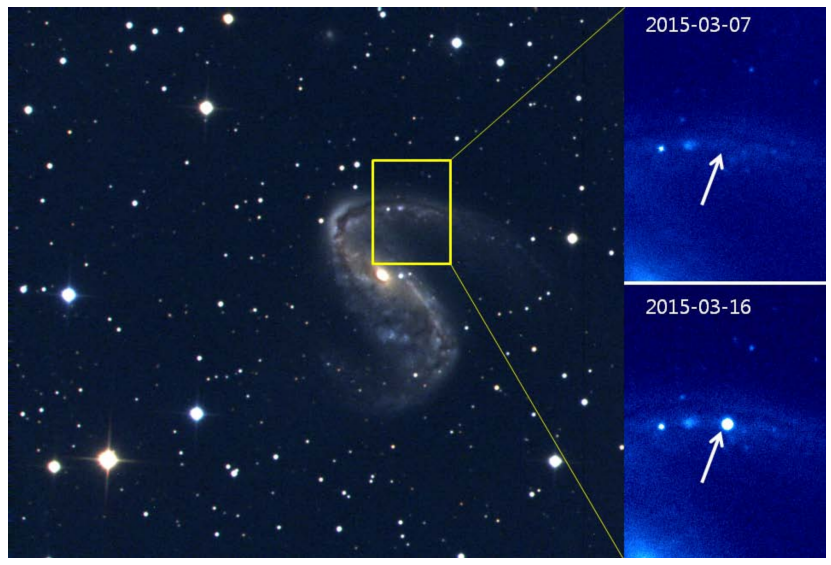

Figure 8. BVR composite image of NGC 2442 and the location of SN $2015 \mathrm{~F}$ before and after its emergence ( $R$-band).

\section{FUTURE UPGRADES}

We hope to upgrade the LSGT system in the near future in two areas.

First, we will work on the telescope optics to improve the image quality. Currently, we mostly achieve image PSF FWHM above 2.'0, as shown in Figure 6. Better image performance has been achieved through the use of other PlaneWave CDK telescopes at the same site. These PlaneWave systems are very similar to LSGT, so there is room for improving the image quality.

Second, we plan to install a new camera and new filters to improve the sensitivity and expand the wavelength coverage. We recently purchased a $1 \mathrm{k} \times 1 \mathrm{k}$ CCD camera (iKon-M 934 Series) from Andor, Inc. that is equipped with a back-illuminated, deep depletion CCD (BEX2-DD) for better sensitivity at both short and long wavelengths. This camera is similar to the CQUEAN camera (Park et al. 2010), but with an improved sensitivity at short wavelengths. The new camera boasts $\gtrsim 90 \%$ QE from 0.4 to $0.9 \mu \mathrm{m}$, with QE $=30 \%$ at $1 \mu \mathrm{m}$ (Figure 3 ). We will match it with an 18 slot filter wheel that will house a standard set of grizY filters and a suite of medium-band filters with wavelength widths of $50 \mathrm{~nm}$ for detailed studies of the spectral energy distribution of objects such as quasars, GRBs, AGNs, and SNe, and photometric reverberation mapping of AGNs. The expected date of operation of the new camera is in the second half of 2015 .

\section{SUMMARY}

In this paper, we presented the characteristics and the current performance of LSGT, and our plan for future upgrades. LSGT is a 0.43 -meter telescope installed at SSO, Australia that can be operated remotely from the SNU campus in Seoul, Korea. It is currently fully operational, with LSGT activities including the use for observational classes at SNU and a long term research project of monitoring nearby galaxies for understanding the nature of transients. The telescope can reach a limiting magnitude of $R \sim 21.5$ mag at $5-\sigma$ with 15 min exposure under good weather (photometric condi- 


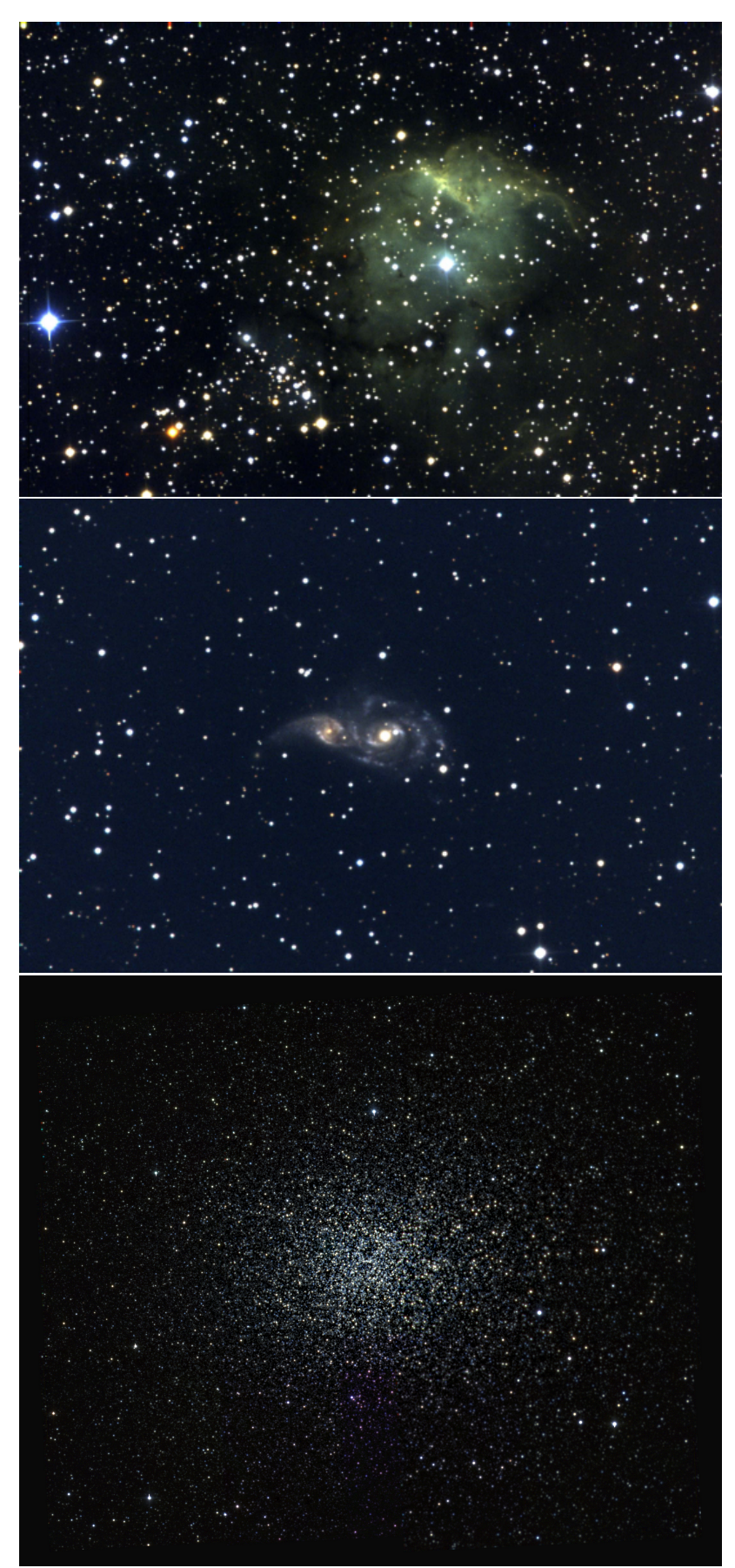

Figure 9. Example LSGT images ( $B V R$ composite). From top to bottom, NGC 2467 ("Skull and Crossbones nebula), NGC 2207 (an interacting galaxy pair), and NGC 5139 (Omega Centauri, a galactic globular cluster). Note that the top two images show a single frame field of view, but the bottom panel is a mosaic of 9 LSGT frames, covering a $30^{\prime}$ by $40^{\prime}$ field. These images were taken with one to three minute exposures.

tion), good seeing (FWHM 2'.2), and dark sky (new moon). In the near future, we plan to improve the optical performance of the telescope and also install a new camera that will be several times more sensitive than the current camera at both short and long wavelengths.

\section{ACKNOWLEDGMENTS}

This work was supported by the Creative Initiative program, No. 2008-0060544, of the National Research Foundation of Korea (NRFK) funded by the Korean government (MSIP). MI acknowledges the hospitality and the support of the Korea Institute for Advanced Study, where part of this work was carried out. This paper includes the data taken at the Siding Spring Observatory in Australia. We thank the staff of iTelescope.Net and SSO, especially Brad Moore, Peter Lake, and Lars Hansen for their professional assistance during the installation, the performance optimization, and the operation of the telescope. We thank Richard Hendick of PlaneWave Instruments for providing us with the telescope efficiency curve. We also thank Sang Gak Lee for her generous contribution to make the telescope possible.

\section{REFERENCES}

Aceituno, J., Sanchez, S. F., Aceituno, F. J., et al. 2011, An All-Sky Transmission Monitor: ASTMON, PASP, 123, 1076

Choi, C., \& Im, M. 2014, SNUO Observation of GRB 130427A, The Tenth Pacific Rim Conference on Stellar Astrophysics, ASP Conference Series, 482, 203

Im, M., Choi, C., Yoon, S.-C., et al. 2015, The Very Early Light Curve of SN 2015F in NGC 2442: A Possible Detection of Shock-Heated Cooling Emission and Constraints on SN Ia Progenitor System, ApJS, submitted

Im, M., Ko, J., Cho, Y., Choi, C., Jeon, Y., Lee, I., \& Ibrahimov, M. 2010, Seoul National University $4 \mathrm{~K} \times 4 \mathrm{~K}$ Camera (SNUCAM) for Maidanak Observatory, JKAS, 43, 75

Kim, E., Park, W.-K., Jeong, H., et al. 2011, Auto-Guiding System for CQUEAN (Camera for QUasars in EArly uNiverse), JKAS, 44, 115

Lee, J., Choe, S.-U., Jung, J.-H., \& Woo, H.-G. 2009, Astronomical Observation Environment Study focusing on Night Sky Brightness Variation under Light Pollution, JKESS, 30, 344

Leinert, C., \& Mattila, K. 1998, Natural Optical Sky Background, Preserving the Astronomical Windows, ASP Conference Series, 139, 17

Lim, J., Chang, S., Pak, S., Kim, Y., Park, W.-K., \& Im, M. 2013, Focal Reducer for CQUEAN (Camera for QUasars in EArly uNiverse), JKAS, 46, 161

Park, W.-K., Pak, S., Im, M., et al. 2012, Camera for Quasars in Early Universe (CQUEAN), PASP, 124, 839

Patat, F. 2003, UBVRI Night Sky Brightness During Sunspot Maximum at ESO-Paranal, A\&A, 400, 1183

Pedani, M. 2009, Sky Surface Brightness at Mount Graham: UBVRI Science Observations with the Large Binocular Telescope, PASP, 121, 778 